\title{
Dispersive hole transport in polymer:carbon nanotube composites
}

\author{
A.R. Inigo, S.J. Henley and S.R.P. Silva \\ Nanoelectronics Center (NEC), Advanced Technology Institute (ATI), \\ University of Surrey, Guildford, GU2 7XH, Surrey, UK.
}

\begin{abstract}
The hole transport properties of poly(2-methoxy, 5-(2`-ethyl-hexoxy)-p-phenylene vinylene) (MEH-PPV) blended with acid oxidized multiwall carbon nanotubes (COOHMWCNT) were investigated in a diode configuration using the time-of-flight (TOF) photocurrent method. While the room temperature hole mobility in pure MEH-PPV films was non-dispersive with positive field dependent mobility, MEH-PPV:COOH-MWCNT blended devices exhibited dispersive transport and negative field dependent mobility. This indicates that the hole mobility in this composite is influenced by positional disorder caused by the presence of COOH-MWCNT in the MEH-PPV matrix. These results strongly suggest that a uniform distribution of $\mathrm{COOH}-$ MWCNT or other inorganic hybrid materials in the organic matrix is important for charge transport in the high mobility nanotubes component to be activated, when used in hybrid or as a substitute to replace lower mobility organic components.
\end{abstract}

\section{Introduction}

The study of Organic materials is a subject of scientific and technological importance due to their ease of processing and flexibility in large area electronics. Among the different types of organic materials, poly $p$-phenylene vinylene (PPV) and its soluble derivatives have excellent 
luminescent properties [1-3]. Among these derivatives, MEH-PPV has been investigated extensively for its potential in display applications. The optoelectronic device characteristics of MEH-PPV are dependent on morphology which is controlled by its processing conditions. Therefore, control over the charge transport properties such as mobility can be obtained by tailoring its morphology [4-6].

For many organic electronic device application such as light emitting diodes or thin film field effect transistors, high, non-dispersive carrier mobilities (both hole and electron) are desired as they allow higher charge carrier densities and lower dispersion in charge carrier velocities. Unfortunately, for most polymers, including MEH-PPV, hole mobility is much greater than electron mobility. In order to achieve a balanced mobility for both carriers and efficient exciton separation or recombination, effort has been expanded to blend polymers with materials having high electron mobility such as carbon nanotubes (CNT).

Acid oxidation of MWCNT has attracted much attention due to their flexibility in processing such as solubility in water, organic solvents and attractive electronic properties including tailoring of its work function values $[7,8]$. These flexibilities in processing make COOH-MWCNT an attractive candidate to be blended with MEH-PPV. Indeed, COOH MWCNT has been used in organic solar cells [9], light emitting diodes [10], field emission displays [11], flexible displays [12] and printed electronics [13]. In terms of charge transport and mobility research, most work has concentrated on using polymers blended with single walled CNT (SWCNT) and its acid oxidized forms in field effect transistor (FET) configurations [14,15]. It has also been found that the best device performance is heavily dependent on optimized processing conditions such as choice of solvents [4,6,16], drying [17,18], annealing [17-19], etc. which led to the required bulk morphology and crystalline structure [17-19]. Deviations from these optimized structures results in poor device performance. It is still a challenge to increase the 
mobility in organic materials by the addition of MWCNT or SWCNT while retaining the electrooptical properties of organic materials. To investigate this further, we measure the hole transport properties of MEH-PPV: COOH -MWCNT blended devices by using the time of flight (TOF) photocurrent technique. The advantage of this technique over FET measurements is that the mobilities measured are more directly related to the mobilities expected in actual solar cell or diode performance.

In $\mathrm{TOF}$, the sheet of charge carriers produced by a short light pulse, moves with a constant velocity in the material under the influence of an externally applied voltage. Departure from a standard transit time across the device, $\mathrm{t}_{\tau}$, for a specific material can be used to understand the charge carrier transport properties. The mobility $(\mu)$ is given by $\mu=\mathrm{L} / \mathrm{t}_{\tau} \mathrm{E}$, where $\mathrm{L}$ is the thickness of the sample and E is the external applied electric field. Ideally one would expect a photocurrent transient of rectangular shape, but in normal experimental conditions this is not observed due to various reasons including charge trapping or material inhomogeneity. The deviation from the ideal shape provides microscopic information about charge transport [20]. In general, the electric field dependent mobility for polymers and organic molecules follow PooleFrenkel behavior [21],

$$
\ln \left(\mu_{(\mathrm{E})} / \mu_{(\mathrm{E}=0)}\right)=\mathrm{SE}^{1 / 2}
$$

Where, $S$ is the slope of the field dependence and $\mu_{\mathrm{E}=0}$ is the zero-field mobility.

\section{Experimental}

MWCNT from Sigma Aldrich were acid oxidized using a general procedure with 3:1 mixture of sulfuric and nitric acid (Fisher Scientific AR grade) at $130^{\circ} \mathrm{C}$ for one hour. These $\mathrm{COOH}-\mathrm{MWCNT}$ was soluble in water and used as a stock solution. The required quantity of 
COOH-MWCNT was obtained by temperature assisted vacuum drying. This dried COOHMWCNT (0.2 and $2 \mathrm{wt} \%)$ was mixed with $5 \mathrm{mg} / \mathrm{ml}$ solutions of MEH-PPV in chlorobenzene (CB). Thick films were prepared by drop-casting MEH-PPV or MEH-PPV:COOH-MWCNT solutions onto ITO coated glass. The ITO was pre-cleaned by treating with organic solvents in the ultrasonic bath and finally with acetone and an oxygen plasma. The coated films were dried for 12 hours at room temperature, under a solvent-rich environment and the residual solvent removed under dynamic vacuum for another 12 hours. Subsequently, the sample was placed in a vacuum chamber and $100 \mathrm{~nm}$ thick top Au contact was thermally evaporated through a shadow mask, to yield an active area of $4 \mathrm{~mm}^{2}$. The thickness of the polymer sample was measured by an Alpha step profilemeter as $2.35 \mu \mathrm{m}$. MEH-PPV and MEH-PPV:COOH-MWCNT devices were prepared together to maintain the same processing conditions. For TOF measurements, a $5 \mathrm{~ns}$ pulse at $532 \mathrm{~nm}$ from YAG laser (Quantel, Ultra, Big Sky Laser) was used as an optical excitation. The photocurrent transients were measured using a $1 \mathrm{GHz}$ Agilent digital storage oscilloscope. The sample was mounted in a vacuum cryostat, and kept under vacuum for 12 hours prior to the measurements. The time resolution of the experiment was limited at short transit times by the RC time constant of the circuit at high temperatures and the dielectric breakdown at high fields. The signal to noise ratio is the limiting factor for obtaining data at low applied electric fields. In order to ensure that the electric field is not perturbed, the total charge $(\mathrm{Q}=\mathrm{CV})$ injected in to the film was kept around $0.01 \mathrm{CV}$, where $\mathrm{Q}$ is the total charge, $\mathrm{C}$ is the structure capacitance and $\mathrm{V}$ is the applied voltage, by adjusting the laser intensity [6]. For example, for a device with capacitance of $30 \mathrm{pF}$, the total charge of $1500 \mathrm{pC}$ can be estimated by using $\mathrm{Q}=\mathrm{CV}$ at the applied voltage of $50 \mathrm{~V}$. To ensure that the photo injected charges do not perturb the internal electric field, they are kept around $\sim 100 \mathrm{pC}$ by adjusting the incident laser intensity [6]. The intersection of asymptotes to the plateau and the declining slope of the current 
transient in the log-log plot was used to determine the transit times. Surface topography was measured by atomic force microscopy (AFM) tapping mode in a Dimension 3100 AFM. Scanning electron microscopy (SEM) images were recorded in a FEI, Quanta 200F.

\section{Results}

Figure 1a shows the TOF transient at room-temperature for MEH-PPV at different applied voltages. They are characterized by an initial spike which is due to the RC time constant of the circuit, a well-resolved plateau followed by a long tail. This well resolved indicates nondispersive transport. The non-dispersive behavior of the reference MEH-PPV devices is characteristic of MEH-PPV devices of high quality with low defect densities and low structural disorders [19,22,23]. However, for the MEH-PPV:COOH-MWCNT devices (Figure 1b) the transient pulse was highly-dispersive.. This type of highly dispersive transport has been observed previously on MEH-PPV with high defect densities or high structural disorder. In this case the possibility of defect densities originating from MEH-PPV can be ruled out, as the MEH-PPV reference devices show non-dispersive charge transport similar to that reported in the literature $[6,16]$. We conclude that the appearance of highly dispersive hole-transport in blended devices is likely due to structural distortion of MEH-PPV packing in the device structure due to the presence of COOH-MWCNT.

For clarity, we have chosen only the current transients at $90 \mathrm{~V}$ from figure $1 \mathrm{a}$ and figure $1 \mathrm{~b}$ to display in figure $1 \mathrm{c}$ in $\log -\log$ scale. The transit times of $0.4 \mathrm{~ms}$ and $5.8 \mathrm{~ms}$ were obtained at the intersection of asymptotes to the plateau and the declining slope for the reference MEH-PPV and MEH-PPV:COOH-MWCNT devices, respectively. The transit time of the MEHPPV:COOH-MWCNT is higher than reference MEH-PPV devices. These results differ from earlier reports of semiconducting polymers such as MEH-PPV blended with SWCNT. SWCNT 
blended with semiconducting polymers exhibited higher mobility than their pristine counterparts [14]. This may provide an indication that blending of COOH-MWCNT with MEH-PPV is not providing the required charge transport network formed by SWCNT as reported earlier.

The calculated mobilities of reference MEH-PPV and MEH-PPV:COOH-MWCNT devices at different applied voltages were plotted in Figure 2. The mobility of MEH-PPV:COOHMWCNT devices were one order magnitude lower than the reference MEH-PPV devices. For example at $70 \mathrm{~V}$, the calculated mobility values are $1 \times 10^{-6} \mathrm{~cm}^{2} / \mathrm{Vs}$ (Electric field $=3.6 \times 10^{5}$ $\mathrm{V} / \mathrm{cm}$ ) and $1 \times 10^{-7} \mathrm{~cm}^{2} / \mathrm{Vs}$ (Electric field $=3.6 \times 10^{5} \mathrm{~V} / \mathrm{cm}$ ) for reference MEH-PPV and MEHPPV:COOH-MWCNT devices respectively. It should be also noted here that the difference between the two devices is field dependent due to the fact that the reference MEH-PPV and MEH-PPV:COOH-MWCNT devices have positive and negative field dependence of mobility respectively.

The field dependent mobility of all devices exhibits a linear relationship according to the Poole-Frenkel relation (Equation 1). Poole-Frenkel behaviour is attributed to the charge transport through a Gaussian density of states in the presence of position and energy disorder as proposed by the Gaussian disorder model (GDM) [20]. According to the GDM, the charge carrier transport in polymer materials is dominated by hopping processes which are affected by two parameters viz. energy disorder and position disorder (structural disorder). Energy disorder is characterized by the variation in the energy levels of hopping sites and the position disorder is characterized by the variation in position and alignment of the hopping sites [20]. These variations mainly arise due to different processing conditions [16].

In summary the experimental results indicate, the hole mobility in the reference MEHPPV devices has a positive field dependence. This indicates low levels of position disorder and is consistent with the observation of the non-dispersive hole transient characteristic of polymers 
devices with lower positional disorder. In contrast, the mobility in MEH-PPV:COOH-MWCNT devices decreases with increasing applied field, i.e., exhibits a negative field dependence. This, along with the highly dispersive nature of hole-transient, is characteristic of devices with an increased position disorder.

Figure 3 shows the surface topography of the MEH-PPV:COOH-MWCNT samples measured by tapping AFM. The AFM images show that the COOH-MWCNT:MEH-PPV devices have higher surface roughness $(\sim 22 \mathrm{~nm})$ than that of reference MEH-PPV devices (roughness $\sim 6$ $\mathrm{nm}$ ). The SEM images (figure 4) also shows that COOH-MWCNT:MEH-PPV devices (Figure 4 right) have more clumps visible on the surface, while the MEH-PPV (Figure 4 left ) devices were smoother and did not show any surface features, at the resolution of the SEM. This figure was included for comparison with the right hand image, which shows surface features that appear (which were not observable in AFM), under very similar SEM imaging conditions, when CNTs are included. The SEM image (figure 4 right) also suggests that the COOH-MWCNT does not distribute evenly and form clusters. These surface studies suggest that the COOH-MWCNT blended with MEH-PPV not only distribute themselves non-uniformly, but may also inhibit the inherent preferentially orientated multidomain formation in MEH-PPV [19]. This clump formation of MWCNT in MEH-PPV matrix may be promoted due to the slow evaporation of solvents in the solvent rich atmosphere used during the device preparation. This slow evaporation is needed for a high quality, higher mobility MEH-PPV film [16].

\section{Discussion}

Variation in morphology is one of the major factors affecting charge transport in conjugated polymers, which depends critically on the sample processing conditions. Since the samples we have prepared are under the same processing conditions except when blending of 
$\mathrm{COOH}-\mathrm{MWCNT}$, the variation in morphology is due to the addition of COOH-MWCNT. Earlier reports suggest that MEH-PPV formed micro domains composed of nano domain structures [19]. These micro domains with nanostructures tend to align along the surface normal. The ratio of domains with preferential orientation along the surface normal were found to vary with processing conditions such as solvent, concentration, thickness and annealing temperature. This spatial variation causes differential hopping rates, and hence dispersion in the velocity of the carriers moving towards the collector electrodes which were excited by a light pulse at the transparent positive electrode. Thus, inhomogeneity in the arrangement of micro domain regions may be a limiting factor for the non-dispersive transport. This conclusion is supported by the observation of the dispersive hole transient, in the case of annealed films, which is an extreme case of inhomogeneous distribution of aggregated and non-aggregated regions [19]. Thus, these inhomogeneities in the film structure may lead to an overall increase in the level of position disorder and affect the type of transport i.e. dispersive or non-dispersive in polymer films significantly.

In addition to the dispersive nature, negative field dependence mobility in MEHPPV:COOH-MWCNT (Figure 2) is also observed. Negative field dependent mobility was reported earlier due to the presence of large position disorder in molecularly doped polymers [24] and in structurally ordered regio regular poly(3-hexylthiophene) (P3HT) [25]. In the first case, position disorder was created due to the doping in molecularly doped polymers, while in the second, it was due to the anisotropic ordering in molecular structures. In the case of molecularly doped polymers, the higher positional disorder provided energetically favorable paths against electric field direction. Hence, the charge carriers travelled longer distances prior to reaching the counter electrode, leading to a longer transit time and subsequently lower mobilities with respect to the applied electric field. This suggests that the highly dispersive transport and weak field 
dependent mobility in MEH-PPV:COOH-MWCNT results from the presence of inhomogeneous distribution and/or orientation of micro domains in MEH-PPV:COOH-MWCNT composites. Such inhomogeneity may lead to a broad distribution of hopping rates and detour of charge carriers against the field direction. In the present case, it appears that the charge carriers may have to move/transfer against the field direction due to an absence of preferentially oriented domains towards the surface normal in MEH-PPV:COOH-MWCNT composites $[19,16]$. The electron current transients were highly dispersive and the transient time was not detectable even on a log-log scale [26]. TOF hole and electron transients from single walled carbon nanotubes blended with poly-N-vinylcarbazole have been reported to be highly dispersive and the $\sim 2$ order of magnitude increase in the mobility attributed to the formation of efficient charge transport networks [27]. As seen in Fig. 2, we do not see any significant increase in the hole mobility even when the concentration is increased from $0.2 \mathrm{wt} \%$ to $2 \mathrm{wt} \%$. Devices with COOH-MWCNT concentrations of $2 \mathrm{wt} \%$ or higher do not have significant change in TOF transients other than the occasional short-circuit observed in some devices due to clumping of MWCNT in the film. This could be due to there being no effective $\mathrm{COOH}-\mathrm{MWCNT}$ network in the blended devices. The increased quantities of $\mathrm{COOH}-\mathrm{MWCNT}$ may increase the chances of clumps formation instead of a $\mathrm{COOH}-\mathrm{MWCNT}$ network.

Figure 5 is the schematic diagram of polymer:CNT blend system, where the charge carrier is transported through three different routes. Type 1: through only the polymer network, Type 2 : through the polymer:CNT network, and Type 3: through the CNT path alone. Very low and high concentrations of CNT with percolated networks could lead to Type 1 and 3 conduction mechanisms respectively. Type 3 may occur for concentrations higher than the percolation concentration threshold. Type 2 conductivity is preferred for utilizing the high mobility of CNTs to replace the lower mobilities of semiconducting polymers in CNT polymer network. On the 
whole, it appears that the MEH-PPV:COOH-MWCNT devices follow type 1 conductivity due to the COOH-MWCNT clumps.

Any method to restore ordering in the packing of the reference MEH-PPV molecules disrupted by the presence of COOH-MWCNT would be of interest. We already observed that annealing would modify the domain structure, but it would produce non-oriented grains (and highly dispersive transport) $[19,22]$. Thus, we believe that thermal annealing would not be a suitable mechanism for removing the disorder introduced by the presence of the MWCNT. $\underline{\text { Hence, }}$ detailed analysis of structural studies with respect to processing on MEH-PPV:COOHMWCNT may provide further understanding and lead the way to effectively utilize the high mobility values of CNT in organic optoelectronic devices.

\section{Conclusion}

In summary, we have prepared MEH-PPV:COOH-MWCNT polymer composite devices by drop casting method. The hole transport in these MEH-PPV thick films by TOF method shows that the observed non-dispersive transport becomes highly dispersive in MEH-PPV:COOHMWCNT devices. The inhomogeneity in the arrangement of micro-domains due to the presence of $\mathrm{COOH}-\mathrm{MWCNT}$ clumps may be a limiting factor in achieving non-dispersive transport and higher charge carrier mobilities.

\section{Acknowledgement}

This research is supported by E.ON AG, as part of the E.ON International Research Initiative. Responsibility for the content of this publication lies with the authors. 


\section{References}

[1] Buroughes J.H, Bradley D D C, Brown A.R, Marks R. N, Mackey K, Friend R.H, Burn R.L and Holmes A.B 1990 Light emitting diodes based on conjugated polymers Nature (London) 347, 539 - 541

[2] Friend R.H, Gymer R.W, Holmes A.B, Burroughes J.H, Marks R.N, Taliani C, Bradley D.D.C, DosSantos D.A, Bredas J.L, Lögdlung M and Salaneck W.R 1999 Nature (London), Electroluminescence in conjugated polymers 397121 - 128

[3] Forero S, Nguyen P.H, Brutting W and Schwoerer M 1999 Charge carrier transport in poly(p-pheylenevinylene) light emitting devices Phys. Chem. Chem. Phys., 11769 - 1776

[4] Liu J, Shi Y, Ma L and Yang Y 2000 Device performance and polymer morphology in polymer light emitting diodes: The control of device electrical properties and metal/polymer contact J. Appl. Phys., 88605 - 609

[5] Nguyen T.Q, Martini I.B, Liu J and Schwartz B.J 2000 Controlling interchain interactions in conjugated polymers: The effects of chain morphology on exciton-exciton annihilation and aggregation in MEH-PPV films J. Phys. Chem. B, 104237 - 255

[6] Inigo A.R, Tan C.H, Fann W.S, Huang Y.S, Perng G.Y and Chen S.A 2005 Enhanced hole mobility in poly-(2-methoxy-5-(2 '-ethylhexoxy)-1,4-phenylenevinylene) by elimination of nanometer-sized domains Adv. Mater., 17, 1835 - 1838

[7] Heister E, Lamprecht C, Neves V, Tilmaciu C, Datas L, Flahaut E, Soula B, Hinterdorfer P, Coley H.M, Silva S.R.P and NcFadden J 2010 Higher Dispersion Efficacy of Functionalized Carbon Nanotubes in Chemical and Biological Environments ACS nano 4 $2615-2626$

[8] Blanchard N.P, Hatton R.A and Silva S.R.P 2007 Tuning the work function of surface oxidised multi-wall carbon nanotubes via cation exchange Chem. Phys. Lett., 43492 - 95 
[9] Hatton R.A, Blanchard N.P, Tan L.W, Latini G, Cacialli F and Silva S.R.P 2009 Oxidised carbon nanotubes as solution processable, high work function hole-extraction layers for organic solar cells Org. Electron., 10388 - 395

[10] Liu D, Fina M, Guo J, Chen X, Liu G, Johnson S.G and Mao S.S 2009 Organic lightemitting diodes with carbon nanotube cathode-organic interface layer Appl. Phys. Lett., 94 $013110-013110-3$

[11] Poa C.H, Henley S.J, Chen G.Y, Adikaari A.A.D.T, Giusca C.E and Silva SRP 2005 Growth and field emission properties of vertically aligned carbon nanofibers $J$. Appl. Phys., 97114308 - 114308-6

[12] Byrne M.T, Gun'ko Y.K 2010 Recent Advances in Research on Carbon NanotubePolymer Composites Adv. Mater., 221672 - 1688

[13] Hsieh G.W, Li F.M, Beecher P, Nathan A, Wu Y.L, Ong B.S, Milne W.I 2009 High performance nanocomposite thin film transistors with bilayer carbon nanotubepolythiophene active channel by ink-jet printing J. Appl. Phys., 106, 123706 - 123706-7

[14] Lee K.Y, Lee S.P, Choi H, Mo K.H, Jang J.W, Kweon H and Lee C.E 2007 Enhanced electroluminescence in polymer-nanotube composites Appl. Phys. Lett., 91023110 023110-3

[15] Bo X.Z, lee C.Y, Strano M.S, Goldfinger M, Nuckolls C and Blanchet G.B 2005 Carbon nanotubes-semiconductor networks for organic electronics: The pickup stick transistor Appl. Phys. Lett., 86182102 - 182102-3

[16] Inigo A.R, Chiu H.C, Fann W.S, Huang Y.S, Jeng U.S, Lin T.L, Hsu C.H, Peng K.Y and Chen S.A 2004 Disorder controlled hole transport in MEH-PPV Phys. Rev. B69 075201 $075201-8$ 
[17] Li G, Shrotriya V, Huang J, Yao Y, Moriarty T, Emery K and Yang Y 2005 Highefficiency solution processable polymer photovoltaic cells by self-organization of polymer blends Nature Mater. 4864 - 868

[18] Ma W, Yang C, Gong X, Lee K, Heeger A.J 2005 Thermally Stable, Efficient polymer solar cells with nanoscale control of the interpenetrating network morphology Adv. Funct. Mater. 15 1617-1622

[19] Jeng U.S, Hsu C.H, Sheu H.S, Lee H.Y, Inigo A.R, Chiu H.C, Fann W.S, Chen S.H, Su A.C, Lin T.L, Peng K.Y, and Chen S.A 2005 Morphology and charge transport in poly(2methoxy-5-(2 '-ethylhexyloxy)-1,4-phenylenevinylene) films Macromolecules, 386566 6574

[20] Bässler H 1993 Charge transport in disordered organic photoconductors - A monte-carlo simulation study Phys. Status Solidi B, 175, 15 - 56

[21] Dunlap D.E 1995 Explanation for the root-dependent mobilities of charge transport in molecularly doped polymers Phys. Rev. B, 52939 - 954

[22] Tan C.H, Inigo A.R, Hsu J.H, Fann W.S and Wei P.K 2001 Mesoscale structures in luminescent conjugated polymer thin films studied by near-field scanning optical microscopy J. Phys. and Chem. of Solids 621643 - 1654

[23] Inigo A.R, Chiu H.C, Fann W.S, Huang Y.S, Jeng U.S, Hsu C.H, Peng K.Y, Chen S.A 2003 Structure and charge transport properties in MEH-PPV Synth. Met., 139581 - 584

[24] Kageyama H, Ohnishi K, Noumura S and Shirota Y 1997 Negative electric-field dependence of hole drift mobility for a molecular glass of tri(o-terphenyl-4-yl)amine Chem Phys. Lett., 277137 - 141

[25] Mozer A.J and Sariciftci N.S 2004 Negative electric field dependence of charge carrier drift mobility in conjugated, semiconducting polymers Chem. Phys. Lett., 389438 - 442 
[26] Scher H and Montroll E.W 1975 Anomalous transit-time dispersion in amorphous solids Phys. Rev. B, 12, $2455-2477$

[27] Tameev A.R, Jimenez L.L, Pereshivko L.Y, Rychwalski R.W and Vannikov A.V 2007 Charge Carrier Mobility in Films of Carbon-Nanotube- Polymer Composites J Phys: Conference Series 611152 - 1156 


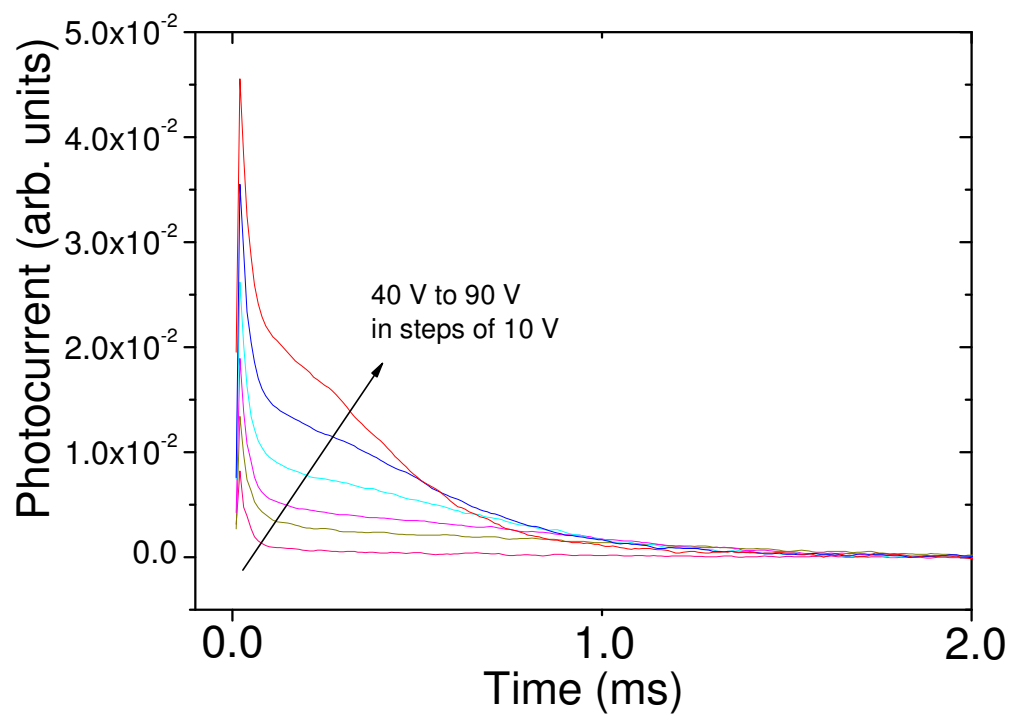

Figure 1a. The non dispersive photocurrent transients at room temperature for the applied voltages from $40 \mathrm{~V}$ to $60 \mathrm{~V}$ in steps of $10 \mathrm{~V}$ for reference MEH-PPV device with thickness of $2.35 \mu \mathrm{m}$. 


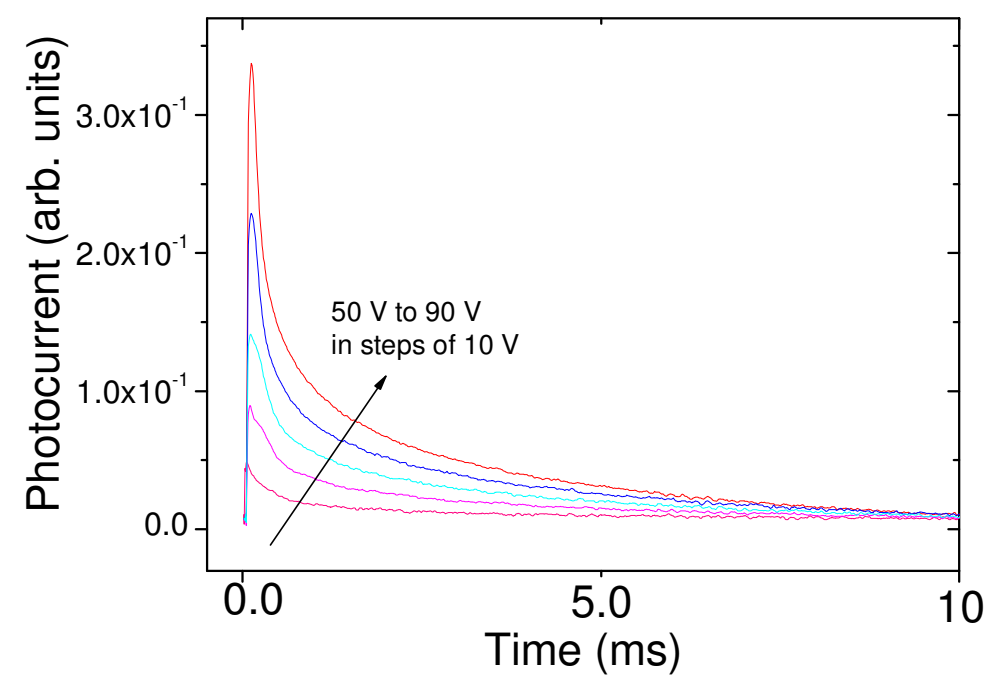

Figure $1 \mathrm{~b}$. The highly dispersive photocurrent transients at room temperature for the applied voltages from $50 \mathrm{~V}$ to $90 \mathrm{~V}$ in steps of $10 \mathrm{~V}$ for MEH-PPV:COOH-MWCNT device with thickness of $2.35 \mu \mathrm{m}$ 


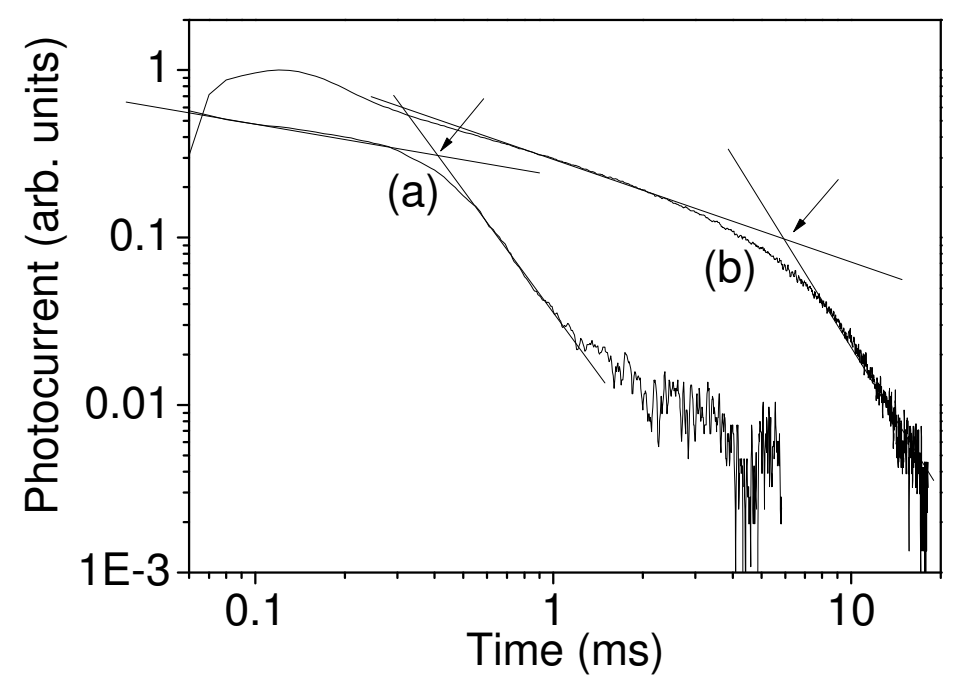

Figure 1c. The representative photocurrents in log-log plot at $90 \mathrm{~V}$ for (a) reference MEH-PPV and (b) MEH-PPV:COOH-MWCNT devices (taken from figure 1a and figure 1b). The arrow shows transit time at the intersection of asymptotes where the plateau and the declining slope of the current transient intersects. 


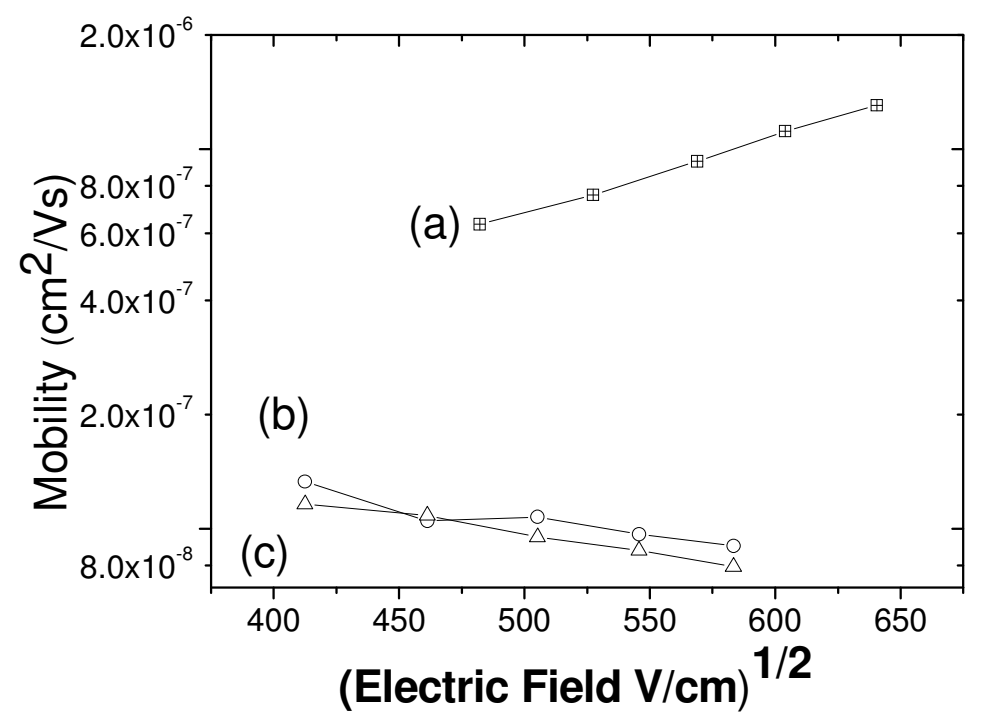

Figure 2. The field dependent mobility at room temperatures for reference (a) MEH-PPV (b) MEH-PPV:0.2 wt\% COOH-MWCNT and (c) MEH-PPV:2 wt\% COOH-MWCNT. 

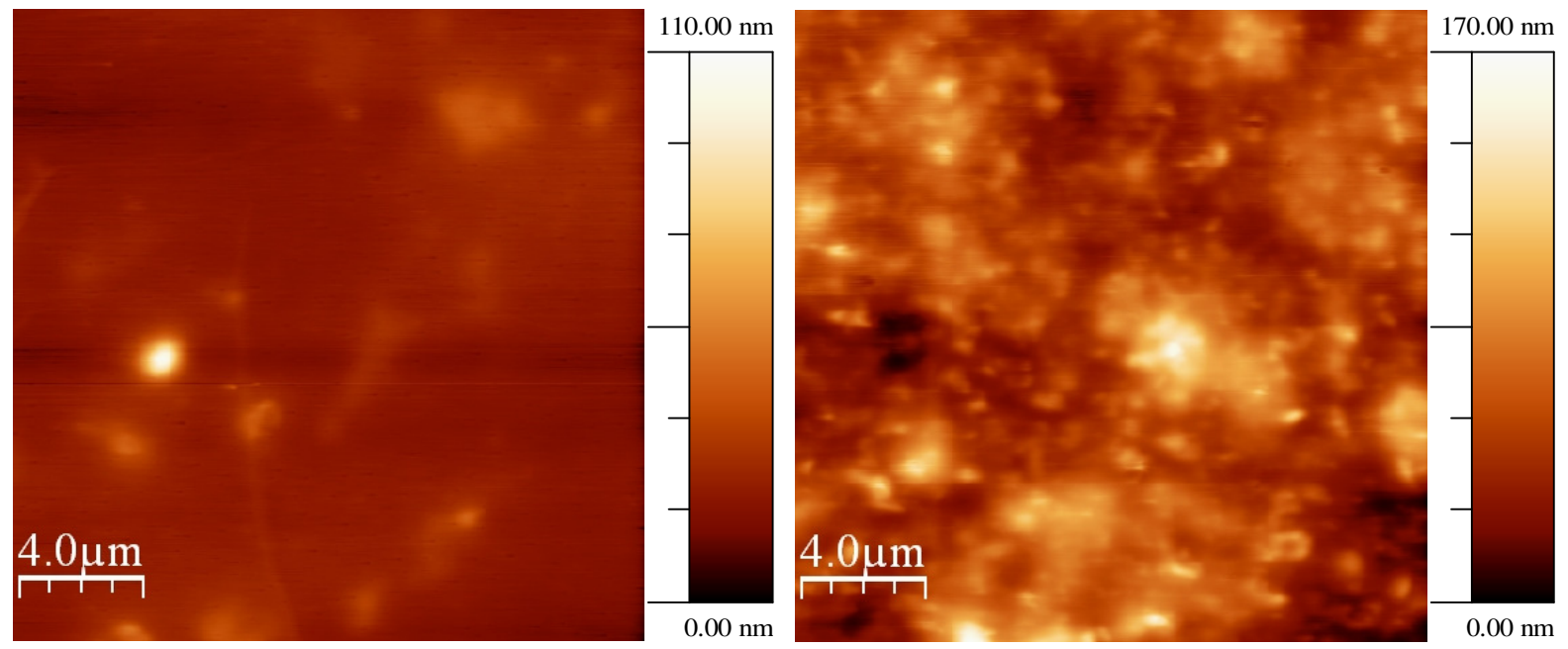

Figure 3. The AFM images of MEH-PPV (left) and MEH-PPV:2 wt\% COOH-MWCNT (Right) with the surface roughness of $\sim 6 \mathrm{~nm}$ and $\sim 22 \mathrm{~nm}$ respectively. While the addition of $\mathrm{COOH}-$ MWCNT makes the surface roughness higher, the COOH.MWCNT clumps were not observed as opposed to that of in SEM (Figure 5). This indicates that the COOH-MWCNT clumps are wrapped within the MEH-PPV polymer matrix. 


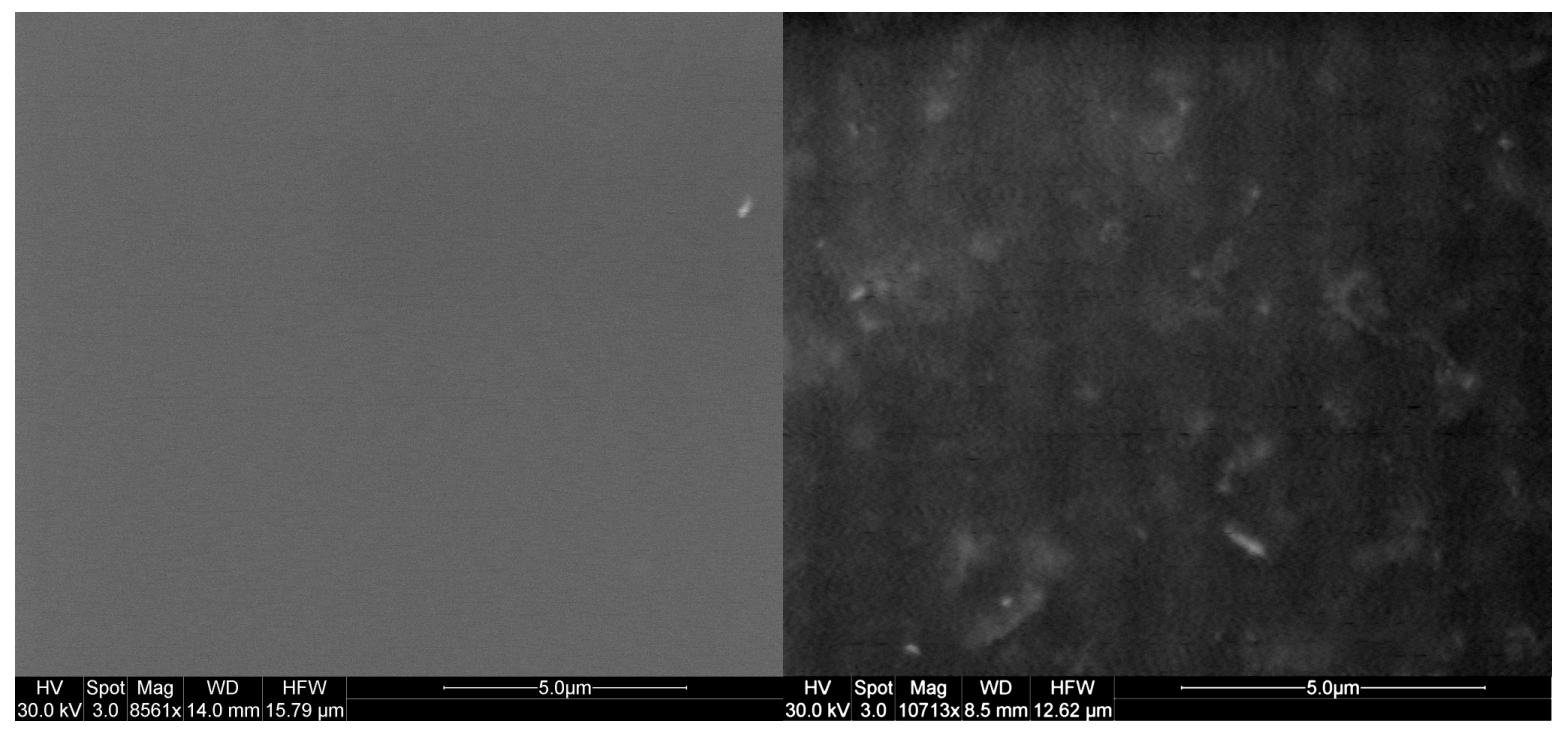

Figure 4. The SEM images of MEH-PPV (left) and MEH-PPV:2 wt\%COOH-MWCNT (Right). The image of high surface quality reference MEH-PPV device shows no significant characteristic features which is consistent with that of low AFM images with low surface roughness. The MEH-PPV:COOH:MWCNT blend films show clumps of COOH:MWCNT (which were not observable in AFM). This disorder in the structures explain the reason for decreased mobility in the MEH-PPV:COOH:MWCNT devices. 

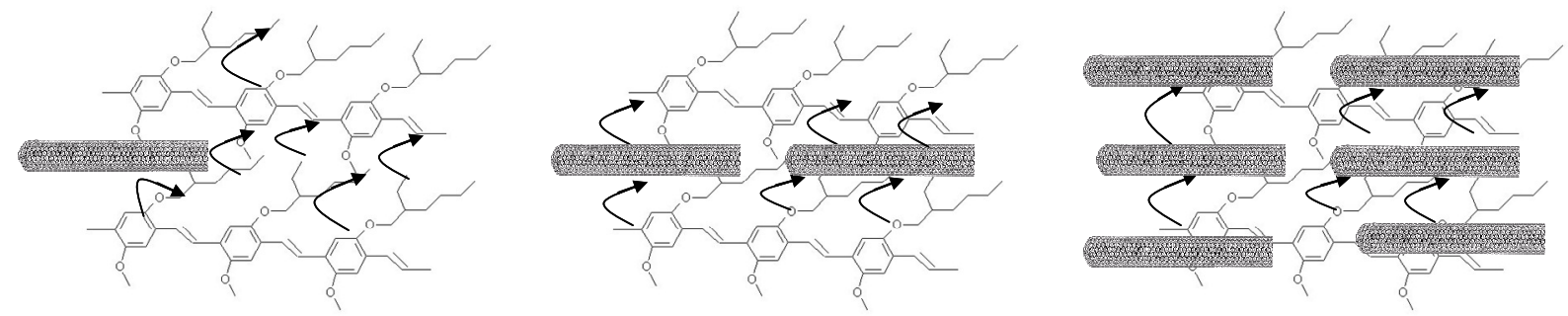

Figure 5. Possible charge carrier pathways (a) Type 1: Charge carrier hopping only through polymer chains, (b) Type 2: charge carrier hopping between polymer:CNT:polymer and (c) Type3: hopping along CNT networks only. 